\title{
Criminologie
}

\section{La peur du crime et la punitivité chez les personnes âgées}

\section{Yves Brillon}

Volume 16, numéro 1, 1983

La peur du crime

URI : https://id.erudit.org/iderudit/017169ar

DOI : https://doi.org/10.7202/017169ar

Aller au sommaire du numéro

Éditeur(s)

Les Presses de l'Université de Montréal

ISSN

0316-0041 (imprimé)

1492-1367 (numérique)

Découvrir la revue

Citer cet article

Brillon, Y. (1983). La peur du crime et la punitivité chez les personnes âgées.

Criminologie, 16(1), 7-29. https://doi.org/10.7202/017169ar d'utilisation que vous pouvez consulter en ligne.

https://apropos.erudit.org/fr/usagers/politique-dutilisation/ 


\section{LA PEUR DU CRIME ET LA PUNITIVITÉ CHEZ LES PERSONNES ÂGÉES Yves Brillon*}

"It is not enough to add years to life, but we must add life to years. :

John F. Kennedy

\section{LA PEUR : DÉBUT DE LA SAGESSE OU DE LA PUNITIVITÉ}

Depuis la fin des années 60 , les recherches portant sur les attitudes et les opinions que manifeste le public, soit envers le phénomène criminel, soit envers le système de justice pénale, se sont multipliées à un rythme inflationniste; cela à un tel point que, comme le dit Kegels, " le crime apparaît aujourd'hui en tête des hit-parades des sondages d'opinion " (1982, p. 210). Cependant, un tel engouement pour les études faites auprès des populations n'est ni fortuit, ni inopportun. En effet, c'est en grande partie l'incapacité, manifestée par les agences pénales, de débusquer la totalité des crimes qui a incité les chercheurs à s'adresser directement aux citoyens. En agissant de la sorte, les chercheurs étaient en mesure :

1. de mieux évaluer la nature et l'importance de la criminalité réelle ;

2. d'identifier l'incidence du phénomène criminel et de la victimisation sur les attitudes des individus et sur leurs comportements;

3. de déterminer les facteurs qui découragent certaines personnes de dénoncer à la police les actes dont elles sont victimes ;

4. d'analyser les attitudes du public autant à l'égard de la justice qu'envers ies objectifs et le fonctionnement des différentes agences pénales.

Les travaux entrepris, aux États-Unis, par la President's Commission on Law Enforcement and Administration (Katzenbach, 1967) ainsi que ceux que poursuit, depuis 1972, le Bureau des statistiques américain pour le Law Enforcement Assistance Administration (L.E.A.A.), ont fourni des indications extrêmement utiles sur l'importance de la victimisation et sur les conséquences qu'elle engendre dans la population, notamment la peur du crime. Dès lors, une myriade de sondages d'opinion ont montré que, un peu partout dans les pays industrialisés, le crime était devenu une des principales préoccupations des citoyens et même, pour une proportion non négligeable d'entre Montréal.

* Chercheur au Centre international de criminologie comparée, Université de 
eux, une source d'angoisse et de peur. On pourrait presque dire, à la limite, que cette peur s'est mutée, s'est transfigurée au point de devenir un objet en soi, qui n'a pratiquement plus besoin du crime comme substrat. On parle de la "peur du crime" comme d'une entité autonome; comme d'une menace per se venue dont on ne sait d'où ; comme d'un mal qui se répand partout et qui, même s'il n'atteint pas tout le monde, du moins ne laisse personne indifférent.

Le rapport Figgie, sur la peur du crime (America Afraid, 1980) affirme, dans son introduction, que " la peur du crime est en train de paralyser lentement la société américaine "(p. 7). Plus loin, il fait, en termes apocalyptiques, l'énumération des effets néfastes de la peur sur la vie sociale : détérioration des villes américaines... ; échec de l'implantation de centres commerciaux dans les petits quartiers...; crainte que nourrissent les individus les uns envers les autres (la peur engendre la méfiance...) ; anxiété vis-à-vis de la possibilité de voir la sécurité de son foyer violée...; évitement, par appréhension, de certaines rues désertes... "La peur du crime de violence, peut-on lire, semble avoir laissé le pays sans secours, incapable de maîtriser les sources de sa peur " (p. 7). Et plus loin, les auteurs du rapport poursuivent dans un langage tout à fait kafkaïen :

Le crime et la possibilité de victimisation sont partout présents, défant le sens de la sécurité individuelle et le sens communautaire du voisinage. Parce qu'il ne semble pas y avoir de moyen pratique pour résoudre le problème, la peur du crime est en train de faire éclater les collectivités en forçant les individus à se réfugier dans un isolement auto-protecteur ; ce qui mine les véritables racines de l'optimisme américain, de la bienveillance entre voisins et de l'aptitude des gens à s'unir dans un effort commun pour accomplir les buts les plus précieux de la société ainsi que pour maintenir les valeurs fondamentales de la communauté démocratique (p. 10).*

Ce discours alarmiste se fonde sur l'augmentation effarante de la criminalité ; augmentation qui serait à l'origine de cette peur qui sape les mécanismes sociaux et qui freine l'essor et le dynamisme de la société américaine. En effet, aux États-Unis, les statistiques montrent un accroissement de $370 \%$ dans le taux de meurtres, entre 1946 et 1973 ; la hausse la plus rapide se situant après 1965. La courbe des vols sérieux est similaire : pendant la même période, elle a subi une poussée ascendante de $300 \%$. En 1978, on comptait un crime de violence contre la personne toutes les 30 secondes et une atteinte à la propriété toutes les 3 secondes (Figgie, 1980). Au Canada, le phénomène criminel

* Traduction libre de l'auteur. 
affiche, comparativement, un profil moins apeurant. Les dernières statistiques criminelles, qui font état de l'évolution de la criminalité entre 1977 et 1981, indiquent qu'il se commet, à l'heure actuelle, un crime de violence toutes les 4 minutes, et un vol toutes les 15 secondes (Statistiques Canada, 1982).

À la vue de ces chiffres et de la façon dont on peut les interpréter, il faut être conscient que les sondages d'opinion peuvent facilement devenir des instruments de manipulation et des véhicules d'idéologie. Il ne faut pas oublier que, fréquemment, la peur du crime est exagérée ou exacerbée par les mass media et exploitée à des fins partisannes par certains groupes, y compris les corps de police, qui peuvent avoir intérêt, en jonglant avec les chiffres et les pourcentages, à voir l'importance du crime s'accroître parce que leur pouvoir et leur prestige en dépendent (Mohr, cité par Brown, 1976). Il n'est pas rare non plus, dans les sociétés industrialisées, que les autorités au pouvoir prennent prétexte d'une recrudescence apparente de la violence pour exiger un plus grand nombre de policiers, d'armes à feu et de gadgets technologiques ainsi que des mesures punitives plus sévères envers les criminels violents (Christie, 1974).

La criminalité soulève des craintes et des implications profondes chez les individus de sorte que les promesses de rétablir l'ordre et la loi et de rendre les rues plus sûres sont des moyens rentables pour s'assurer d'un soutien populaire (Conklin, 1975). Et sous le couvert de ramener l'ordre et la sécurité, les pouvoirs publics peuvent en profiter pour prendre des initiatives (les opérations "coup de poing " en France et de "sécurisation " en Belgique ; l'ouverture du courrier ou l'écoute électronique, etc.,) qui sont des " prétextes "à limiter les libertés, à étendre abusivement le contrôle des personnes, à abolir même certains droits constitutionnellement garantis (Kegels, 1982, p. 212).

Nous tenions à formuler ces mises en garde avant d'aborder la peur du crime chez les personnes âgées, car il s'agit là et d'un sujet et d'un groupe-cible qui offrent facilement prise à l'émotivité. Ce qui risque d'égarer, dans leurs démarches objectives, certains chercheurs et de soulever, dans le public, des réactions irrationnelles.

\section{LES PERSONNES ÂGÉES ET LE CRIME; PLUS DE PEUR QUE DE MAL ?}

Dans le sillage des enquêtes sur les victimes, on en vint, à partir de 1970 , à s'intéresser à la victimisation des personnes âgées. Cet intérêt provenait à la fois des criminologues, qui considéraient l'âge comme une variable qui était liée de façon significative autant à la vic- 
timisation qu'à la peur du crime, et à la fois des gérontologues, qui focalisaient leur attention sur les besoins et les problèmes du vieillissement. Au stade des investigations préliminaires, les affirmations des uns et des autres reposaient beaucoup plus sur des hypothèses que sur des données factuelles.

C'est ainsi que les premières études criminologiques arrivèrent à la conclusion que les personnes âgées étaient plus susceptibles d'être victimes d'actes criminels que les autres groupes d'âge (Butler, 1975; Cunnigham, 1975). Goldsmith et Tomas (1974) affirmaient que la criminalité qui touchait les personnes âgées avait atteint les proportions d'une crise. À quoi Cook et Cook (1976) répondaient que cette pseudo crise n'avait aucun fondement scientifique et qu'elle était plutôt créée et nourrie de toute pièce par les médias d'information. En fait, les criminologues qui voyaient, dans le troisième âge, le groupe le plus victimisé, appuyaient leur argumentation en se référant à des considérations autant quantitatives que qualitatives. Le fait que des facteurs physiques, économiques et environnementaux soient associés au vieillissement contribuait, à leurs yeux, d'une part à accroître les risques de ces personnes à être l'objet d'attaques criminelles et, de l'autre, à magnifier chez elles les incidences de la victimisation (Goldsmith et Goldsmith, 1976). Le Congrès américain, prêtant une oreille attentive aux divers groupes de pression, constitués par des regroupements de personnes âgées, en arriva à soutenir la même affirmation : les personnes âgées, qu'elles habitent des logements privés ou des centres d'accueil publics, sont les personnes qui offrent la plus grande vulnérabilité à l'égard du vol, de la violence, du harcèlement par des voyous (rowdyism) et du terrorisme spontané (outright terrorism) (U.S. Congress, 1972). Cet intérêt gouvernemental pour la qualité de vie des personnes âgées se confirma, dans les faits, par la reconnaissance de la protection policière des personnes âgées comme étant une des plus hautes priorités.

Cette hypothèse d'une victimisation plus grande chez les gens du troisième âge n'a pas survécu à l'épreuve de recherches plus poussées. Des sondages extensifs, sur des échantillons représentatifs, prouvèrent, apparemment hors de tout doute, que les personnes âgées étaient moins victimes de crimes et d'infractions que les autres groupes d'âge (Cook, 1976; Cook \& Cook, 1976). Ce qui amena le U.S. Subcommittee on Housing and Consumer Interests on the Select Committee on Aging à reconnaitre que les taux nationaux de victimisation étaient plus bas pour les personnes âgées que pour la population générale. Toutefois, dans le rapport qu'il publia (In Search of Security : $A$ 
National Perspective on Elderly Crime Victimization, 1977) il souleva l'hypothèse que, même s'il y avait une controverse concernant les mesures quantitatives de la victimisation des personnes âgées, il n'en restait pas moins que ces dernières, selon les témoignages des professionnels entendus en audiences, souffraient - lorsqu'elles étaient victimes d'une façon disproportionnée en comparaison avec le reste de la population.

On passa ainsi de la reconnaissance d'un plus haut taux de victimisation, pour les personnes âgées, à une victimisation plus pénalisante, plus affligeante et plus cruelle. Cette hypothèse s'appuie sur certaines caractéristiques de la population du troisième âge dont, entre autres, la condition physique, l'état financier et la situation matérielle.

\section{a) La condition physique}

Près de $85 \%$ des personnes de 65 ans et plus souffrent d'une ou de plusieurs maladies chroniques, ce qui les rend plus vulnérables (Lawton et al., 1976) et souvent incapables de s'échapper ou de résister à une attaque (Goldsmith et Goldsmith, 1976). De plus, les conséquences d'une blessure mineure peuvent être extrêmement graves. Une bousculade peut entrainer une chute et provoquer une fracture qui pourra être très lente à guérir et même résulter en une invalidité permanente (Logan, 1979 ; Goldsmith et Goldsmith, 1976).

\section{b) L'état financier}

Comparativement aux autres groupes de la population, les citoyens âgés sont ceux qui ont le plus haut taux de pauvreté (Levitan, 1976). Aux États-Unis, parmi les personnes de 65 ans et plus, une sur six vit dans un état de pauvreté contre une personne sur neuf, pour les gens de moins de 65 ans (Cook et al., 1978). La situation serait pire au Québec où selon l'Association québécoise pour la défense des droits des retraités, deux tiers des personnes âgées vivraient sous le seuil de pauvreté (Radio-Canada, émission "Ce Soir" du 6 novembre 1979).

Les revenus fixes des gens du troisième âge s'érodent avec l'inflation ; ce qui amène une paupérisation de plus en plus marquée, un déplacement des vieux vers des quartiers défavorisés où les taux de criminalité et les risques de victimisation sont plus élevés (Grossman, 1977). En même temps, cette mobilité sociale est susceptible de s'accompagner d'une dérivation vers des appartements de moins en moins adéquats et d'occasionner des privations au niveau de la nourriture, de l'achat de vêtement, de médicaments, etc. Dans ces conditions, des 
pertes financières consécutives à des vols ou à des larcins sont relativement importantes pour eux (Hahn, 1976).

\section{c) La situation matérielle}

Les personnes âgées étant sans travail et ne comptant que sur des revenus fixes et limités, les biens matériels dont elles disposent ne sont pas toujours remplaçables. Ce qui fait que le vol constitue, dans bien des cas, la suppression d'un objet que l'on ne pourra plus, ou difficilement, se procurer parce que trop cher (téléviseur, bijou, etc.) ou parce qu'irremplaçable (photographies de famille, souvenirs à valeur affective, etc.). Le vol d'un sac à main et la perte de papiers essentiels cartes d'assurance-maladie, d'autobus, d'identification, etc., - peuvent créer chez des personnes âgées et malades des inconvénients fâcheux et entraîner des démarches fastidieuses (Logan, 1979).

Ces caractéristiques des conditions de vie des personnes âgées tendent à démontrer que celles-ci sont susceptibles de souffrir davantage (physiquement, financièrement et psychologiquement) que les autres lorsqu'elles sont victimes d'un crime ou d'un délit. Or, même cette hypothèse ne trouve pas grâce devant certains criminologues, dont Fay Lomas Cook, Wesley G. Skogan, Thomas D. Cook et George E. Antunes (1978). Ces auteurs, en analysant les données des sondages nationaux américains de 1973 et de 1974, essaient de prouver que les personnes âgées, par rapport aux autres groupes d'âge, ne subissent pas, lorsqu'elles sont victimes, de conséquences physiques plus sévères ou de pertes financières plus grandes.

Leur démonstration, qui se base sur les statistiques, ne tient évidemment pas compte des incidences psychologiques de la victimisation. En étudiant la valeur médiane des pertes subies, lors de vols, selon l'âge du chef de famille, et lors de vols sur la personne, selon l'âge de la victime, ils démontrent que les personnes âgées : (1) sont moins souvent victimes d'un crime que les autres ; (2) que les pertes qu'elles subissent, en chiffres absolus, sont les mêmes ou sont moins élevées que pour les autres adultes; (3) que ces mêmes pertes, en valeurs relatives, c'est-à-dire en fonction du revenu mensuel, sont les mêmes ou sont plus élevées que pour les autres adultes.

C'est en se fondant également sur des séries statistiques qu'ils examinent, aussi, les assauts et leurs conséquences pour les différents groupes d'âge. Ce qui les conduit à conclure que, en ce qui a trait aux blessures, les personnes âgées : (1) sont moins agressées que les autres ; (2) sont plus souvent blessées si elles sont attaquées ; (3) ont moins de 
blessures ou d'os et de dents brisés ; (4) subissent davantage de lésions internes, de pertes de conscience, de coupures et de contusions ; (5) ne nécessitent pas plus que les autres de soins médicaux généraux ; (6) ou de soins médicaux payants; (7) ou de soins entraînant un coût très élevé ; même si (8) les coûts des soins représentent une beaucoup plus grande proportion de leur revenu que pour les autres groupes.

Nous devons admettre que la démarche que poursuivent Cook et al. soulève une certaine irritation. D'abord parce qu'elle est sans nuance, qu'elle est tout entière rivée à des chiffres qui sont interprétés d'une manière qui apparaît tout à la fois restrictive et rigide. Restrictive, parce qu'on ne retient que les interprétations qui soutiennent les postulats des auteurs sans les moduler; rigide, parce que les chiffres sont examinés en eux-mêmes, sans tenir compte de leurs valeurs différentielles.

Or, une perte de $120 \$$ par exemple, chez un jeune homme qui gagne 12,000\$ par année, et qui habite chez ses parents, n'a pas la même signification que pour une femme âgée qu'un revenu identique maintient dans dans un état de pauvreté. Et pour les blessures, l'analyse comparative des auteurs devient quelque peu abusive. Lésion pour lésion, dent brisée pour dent brisée, frais médicaux pour frais médicaux, ce qui fait la différence, pour les groupes d'âge, ce n'est sûrement pas le nombre de blessures mais bien l'âge des victimes. Être pauvre et vieux ; être pauvre et malade, c'est quand même pire que d'être pauvre et en bonne santé ou que d'être pauvre et jeune. Le ton de l'article de Cook et al. s'avère à certains égards polémique dans la mesure où ils cherchent à convaincre les lecteurs qu'une catégorisation des victimes selon le critère de l'âge est sans fondement. En conséquence, à leur avis, il serait préférable d'établir cette catégorisation en fonction du revenu. En d'autres mots, si les personnes âgées se différencient des autres, au niveau de la victimisation, ce n'est pas parce qu'elles sont vieilles mais parce qu'elles sont pauvres (elderly victims are a special group more because of their lower incomes than because of some innate frailty; Cook et al., 1978, p. 349).

Le but poursuivi par les auteurs ne paraît pas désintéressé. Il est motivé, semble-t-il, par le fait que le gouvernement américain réserve des fonds importants pour la protection des personnes âgées et pour l'indemnisation de celles d'entre elles qui sont victimes de crimes. Or, l'argumentation développée dans l'article vise à briser ce cloisonnement de l'aide aux victimes pour la rendre plus universelle. En effet, si l'aide était distribuée en fonction du revenu plutôt qu'en fonction de l'âge, il est certain que plus de citoyens en bénéficieraient (dont les 
Noirs qui sont parmi les plus pauvres et les plus victimisés). Il est évident que cette expansion de l'assistance gouvernementale ne peut être que souhaitable et bénéfique. Mais pour y parvenir, nous ne voyons pas la nécessité de gommer et d'occulter les problèmes spécifiques aux perşonnes âgées en déniant, à lâge et à la vieillesse, leurs caractéristiques propres ainsi que la vulnérabilité qu'ils engendrent.

D'autres chercheurs sont loin de partager les points de vue de Cook et al. (1978) et reconnaissent au vieillissement un élément particulier qui aggrave les effets de la victimisation. Hahn, entre autres, considèrent avec beaucoup d'émotivité ce problème et déclare : (1) qu'il n'existe pas de choses telles que de petits crimes contre les personnes âgées ; et (2) que les victimes âgées devraient être traitées avec la même attention compatissante et devraient déclencher la même mobilisation de ressources communautaires que pour les victimes de désastres naturels. Selon lui, les conséquences du crime sont presque toujours graves pour cette catégorie de la population. Et il résume, en quatre types, les incidences que peut avoir le crime pour les personnes âgées : " (a) des dommages et des souffrances physiques ; (b) des coûts financiers qui, dans beaucoup de cas, sont désastreux ; (c) des traumatismes émotifs, dont spécialement la peur; et (d) des changements dans le style de vie qui, fréquemment, se traduisent par un plus grand retrait, un plus grand isolement et même par la mort " (Hahn, 1976; p. 123).

À l'heure actuelle, comme on a pu le constater, il y a beaucoup d'ambiguités dans les analyses que font les criminologues de la victimisation des personnes âgées. Cette situation découle du manque de recherches spécialement orientées vers les problèmes propres à ce groupe d'âge. La plupart des données sont donc constituées par des études secondaires, effectuées sur les résultats des sondages que mène régulièrement le L.E.A.A. Mettant de côté les divergences de vue, qui découlent le plus souvent de la confrontation entre les méthodologies quantitatives et qualitatives, un certain consensus semble s'être établi sur les points suivants :

1. les personnes âgées sont moins victimes de crimes et de délits que les autres adultes (Skogan, 1980);

2. les personnes âgées ont plus peur du crime que les autres groupes sociaux (Adams \& Smith, 1976) et cette peur semble s'accroître avec le temps.

Ce paradoxe apparent pourrait s'expliquer par l'argumentation suivante : comme les personnes âgées ont plus peur du crime que les 
autres, elles s'isolent davantage, donc elles s'exposent moins au crime ; d'où leur plus bas taux de victimisation.

MOINS VICTIMES DU CRIME, LES PERSONNES ÂGEES LE SERAIENTELLES PLUS DE LA PEUR?

Lindquist et Duke (1982) pensent que, dans la mesure où l'on pourrait pondérer le facteur d' "exposition aux risques", les personnes âgées auraient un taux de victimisation ou bien égal ou bien supérieur à ceux des autres groupes d'âge. Le fait qu'elles se perçoivent comme ayant de fortes probabilités d'être victimes de crimes, même si elles sont moins victimisées, montre qu'elles ont une perception exacte de la réalité (Lawton, 1980). Cette perception entraîne une plus grande peur qui les oblige à restreindre leurs activités (Hahn, 1976). La peur du crime, plus que le crime lui-même, serait ce qui différencierait les gens du troisième âge : "It is reasonable to argue that for the older people fear of crime is even more of a problem than crime itself "(Clemente and Kleiman, 1976, p. 207). Ce postulat reste à être vérifié. Et, également à être nuancé, notamment en précisant de quoi auraient peur les personnes âgées ?

Selon les sondages américains, la peur du crime (mesurée par la crainte de marcher seul le soir dans un rayon d'un mille de chez soi) serait non seulement plus grande pour les groupes d'âge les plus élevés mais s'accroîtrait aussi, avec le temps, davantage que chez les jeunes de 18 à 25 ans. Entre 1965 et 1974, la proportion des personnes âgées qui exprimait de la crainte était de $38 \%$ en 1965 , de $33 \%$ en 1967 , de $41 \%$ en 1968 , de $46 \%$ en 1973 et de $56 \%$ en 1974 . Chez les jeunes, pendant la même période, les pourcentages étaient respectivement de 35 , de 30 , de 37 , de 40 et de $43 \%$. Cook (1980) fait remarquer que ce qui est le plus important, dans ces chiffres, c'est que la différence, entre les jeunes et les vieux, s'est accrue de façon dramatique. En 1965 et en 1966, la différence, dans les pourcentages d'individus se disant craintifs pour chacun de ces groupes d'âge, était d'environ $3 \%$; en 1968, elle passait à $4 \%$; en 1973 , elle se situait à $6 \%$; en 1974 , elle grimpait à $13 \%$. Ainsi, la peur du crime chez les personnes âgées se distancie de plus en plus de celle chez les jeunes adultes.

Par ailleurs, un autre sondage national, celui-là exécuté par Harris en 1974 (voir Cook, 1980), montre que le crime est considéré, par les personnes âgées, comme un problème "très sérieux ", à tel point qu'il arrive en tête de liste de leurs préoccupations. En effet, le pourcentage d'entre elles (23\%) qui qualifient le crime comme "très sé- 
rieux" est plus élevé que celui des personnes qui perçoivent comme " très sérieux " les problèmes de santé $(21 \%)$, les problèmes d'argent $(15 \%)$, les problèmes de solitude $(12 \%)$, ou les problèmes de soins médicaux (10\%). Il semblerait donc admis que la peur d'être victime soit plus élevée chez les personnes âgées que chez les jeunes. Skogan (1978) pense que cela serait dû moins à l'âge qu'au sexe. Selon lui, la concentration disproportionnée de femmes parmi les 65 ans et plus serait un facteur de distorsion qui se trouverait à exagérer la relation positive entre la peur et l'âge.

Il est vrai que tous les Gallup et toutes les enquêtes du L.E.A.A. ont prouvé qu'une proportion substantiellement plus forte de femmes que d'hommes ont peur de marcher seules la nuit dans leur quartier. Entre 1967 et 1977, le pourcentage de femmes craintives est passé de $46 \%$ à $63 \%$. Pendant ce même laps de temps, celui des hommes fluctuait entre 16 et $23 \%$. En gros, aux États-Unis, il y a presque toujours trois fois plus de femmes que d'hommes qui ont peur du crime (Stinchcombe et al., 1980). Une recherche que nous avons effectuée au Canada (G.R.A.C., 1982) ${ }^{3}$ montre que $26 \%$ des personnes interrogées (213 sur 817 répondants) pouvaient être considérées comme craintives, puisqu'elles déclaraient que "souvent " ou " très souvent " elles évitaient de sortir seules le soir parce qu'elles avaient peur du crime. Parmi ces personnes craintives, on dénombrait $84 \%$ de femmes $(\mathrm{N}=179)$ et $16 \%$ d'hommes $(\mathrm{N}=34)$; et, parmi les femmes, on comptait plus de personnes âgées (29\% d'entre elles ayant plus de 61 ans) que chez les hommes (6\% d'entre eux étaient âgés de plus de 61 ans).

Ces données sembleraient accréditer la thèse de Skogan, déjà citée, selon laquelle ce serait le sexe plus que l'âge qui expliquerait la peur chez les personnes âgées. Toutefois, et nous y reviendrons, si le sexe influence la distribution de la peur du crime chez les gens du troisième âge ce n'est pas parce que les femmes sont disproportionnellement plus nombreuses dans les groupes d'âges plus élevés (argument de Skogan) mais bien plutôt parce que les femmes âgées sont disproportionnellement plus craintives que les hommes âgés.

3. Recherche portant sur "Les attitudes du public canadien envers les politiques criminelles n. Enquête pilote, rapport final par Yves Brillon, Christiane Louis-Gérin et Marie-Christine Lamarche. Cette recherche fut faite sur la demande du ministère du Solliciteur général du Canada qui la finança (TB/CT Reg. 23012). L'échantillon " théorique " était constitué par une population fortement urbanisée (Montréal, Toronto et Winnipeg : N 614) et par une population rurale des provinces de Québec, d'Ontario et du Manitoba (N 203). 


\section{CHEZ LES PERSONNES ÂGÉES :PEUR DIFFUSE OU PEUR CONCRÈTE D'ÊTRE VICTIME ?}

Bien que, dans la plupart des recherches, les auteurs ne définissent pas la "peur" de façon explicite, ce qu'ils entendent le plus souvent par ce concept c'est la perception qu'a une personne de ses probabilités d'être l'objet d'un crime ou d'un délit. Par exemple, Sundeen et Mathieu (1966b) donnent de la peur la définition suivante : "C'est le niveau d'anxiété et de préoccupation qu'une personne a d'être victime ", (p. 55). La question classique, dans les enquêtes de victimisation, celle que l'on pose dans les sondages depuis 1948 pour mesurer la peur du crime, est celle dont nous avons étudié, plus haut, certains résultats, à savoir : "Is there any area right around heere - that is, within a mile - where you would be afraid to walk alone at night?".

Cette définition de la peur du crime est beaucoup trop vague et la question sur la crainte de sortir seul le soir laisse beaucoup d'imprécisions sur ce qu'elle mesure réellement. Yin (1980) juge cette définition inadéquate parce qu'elle ne retient qu'un seul élément qui est celui de la probabilité d'être victimisé, que cette probabilité soit imaginaire ou réelle. À la suite de gérontologues, il suggère que l'on ajoute deux éléments pour mieux cerner le sentiment de crainte chez les personnes âgées : la gravité des conséquences physiques et/ou financières encourues par les personnes âgées et leur capacité de se remettre (physiquement ou financièrement) de leur expérience de victimisation. Aucune recherche n'a encore, à notre connaissance, poussé l'analyse aussi loin que Yin ne le voudrait. Toutefois, un pas dans cette direction a été franchi par l'enquête que Harry E. Figgie a dirigée aux États-Unis sur la peur du crime (The Figgie Report on Fear of Crime : America Afraid, 1980) et par la recherche faite au Canada, par la G.R.A.C. (1982).

Le rapport Figgie examine deux dimensions de la peur ; dimensions qu'il identifie comme étant respectivement la "peur concrète" (concrete fear) d'être victime d'actes de violence et la "peur diffuse " (formless fear) d'atteintes non spécifiques à la sécurité. Pour chacun de ces types de peur, un indice a été constitué :

a) Indice de la peur concrète : Il mesure l'intensité, chez une personne, de l'inquiétude vis-à-vis de six crimes majeurs ; c'est-à-dire la peur qu'elle a d'être tuée, d'être agressée sexuellement ou violée, d'être attaquée ou assaillie avec violence, d'être poignardée, d'être battue, d'être victime d'un vol à main armée.

b) Indice de la peur diffuse : Cet indice établit un baromètre de la crainte qu'une personne peut ressentir, face à des menaces non dé- 
terminées, que soulève chez elle son milieu de vie : crainte d'être seule, pendant le jour et pendant la nuit, soit dans le centre-ville ou dans un grand centre d'achat, soit dans son quartier, ou soit encore chez elle.

L'analyse de la distribution de ces indices, selon l'âge, montre que la peur concrète est significativement reliée à l'âge (gamma $=, 17)$ : elle est plus élevée chez les jeunes et elle diminue progressivement chez les personnes plus âgées. Pour ce qui est de la peur diffuse, le lien avec l'âge est moins étroit (gamma $=, 08$ ). Il agit, cependant, en sens inverse, c'est-à-dire que le niveau de peur diffuse est plus élevé chez les plus vieux que chez les plus jeunes. C'est ce qu'illustre le Tableau I.

\section{TABLEAU I}

Impact de l'âge sur la peur concrète et sur la peur diffuse du crime, selon le Rapport Figgie (1980)

\begin{tabular}{|c|c|c|c|c|c|c|c|c|}
\hline & \multicolumn{4}{|c|}{ Peur concrète } & \multicolumn{4}{|c|}{ Peur diffuse } \\
\hline & \multicolumn{2}{|c|}{$\begin{array}{l}\text { Moins de } \\
60 \text { ans }\end{array}$} & \multicolumn{2}{|c|}{$\begin{array}{c}60 \text { ans et } \\
\text { plus }\end{array}$} & \multicolumn{2}{|c|}{$\begin{array}{c}\text { Moins de } \\
60 \text { ans }\end{array}$} & \multicolumn{2}{|c|}{$\begin{array}{c}60 \text { ans et } \\
\text { plus }\end{array}$} \\
\hline & $\mathrm{Nb}$ & $\%$ & $\mathrm{Nb}$ & $\%$ & $\mathrm{Nb}$ & $\%$ & $\mathrm{Nb}$ & $\%$ \\
\hline Peur élevée & 344 & (43) & 78 & (33) & 278 & (35) & 102 & (43) \\
\hline Peur modérée ou faible & 448 & (57) & 158 & (67) & 514 & (65) & 135 & (57) \\
\hline Total & 792 & $(110)$ & 236 & $(100)$ & 792 & $(100)$ & 237 & $(100)$ \\
\hline
\end{tabular}

La peur concrète d'être victime de crimes graves atteint un haut niveau chez $43 \%$ des gens de moins de 60 ans (le score le plus élevé étant de $49 \%$ chez les jeunes de 18 à 29 ans) et chez $33 \%$ des 60 ans et plus. Les femmes $(46 \%)$ plus que les hommes $(34 \%)$ affichent une grande crainte d'être victime de violence. Il semble donc que la peur des crimes violents soit le reflet des probabilités de victimisation réelle, du genre de vie, des caractéristiques socio-démographiques et du degré d'exposition aux risques. Selon le F.B.I. Uniform Crime Report, les individus âgés de 20 à 29 ans fournissent $33 \%$ des victimes de meurtres alors qu'ils ne représentent que $17 \%$ de la population américaine. Par ailleurs, 55\% des femmes ont peur du viol. En 1978, il y a eu 67000 viols aux États-Unis (Figgie, 1980). Les auteurs du Rapport Figgie résument, comme suit, les caractéristiques de la population qui affiche un degré élevé de crainte concrète : ce sont des gens qui habitent les grandes villes, qui sont jeunes, qui sont -pour une grande part - de sexe féminin, qui appartiennent plus souvent aux minorités noires 
et qui, plus que les autres, s'exposent aux informations que diffusent les mass media sur le crime.

C'est la peur diffuse qui semble caractériser davantage les personnes âgées. Dans cette catégorie d'âge (60 ans et plus), on retrouve un pourcentage plus élevé de personnes craintives $(43 \%)$ que pour les autres groupes d'âge $(35 \%)$. Bien que les facteurs sociodémographiques interviennent également ici, notamment le fait de vivre dans une grande ville, d'être Noir et d'être femme, ce qui spécifie cette crainte diffuse c'est qu'elle est influencée par les conditions sociales défavorables (bas revenu, éducation peu poussée) et par l'isolement personnel (veuvage, être sans travail, solitude). Bref, l'insécurité financière, sociale et individuelle engendre, en partie, une insécurité diffuse vis-à-vis de la criminalité.

La recherche qui a été faite au Canada (G.R.A.C., 1982) confirme les résultats de l'enquête d'Harry E. Figgie Jr. Cette recherche distinguait, elle aussi, la "peur concrète " (ou le danger appréhendé d'être victime) de la "peur diffuse" (ou le sentiment d'insécurité face au crime). L'indice de la peur concrète, moins développé que celui du Rapport Figgie, repose sur les risques perçus d'être victime de deux crimes, soit la probabilité : (1) d'être volé chez soi après avoir quitté son domicile, et (2) d'être attaqué chez soi par un inconnu. Quant à l'indice de la peur diffuse, il a été élaboré à partir de cinq éléments : la crainte de fréquenter certains quartiers ; l'évitement de certaines activités par peur du crime; le degré du sentiment d'insécurité ressenti dans son quartier ; la crainte de sortir seul le soir, et le désir de changer de quartier par manque de sécurité.

L'étude des résultats de cette recherche paraît indiquer un impact différentiel de l'âge, selon le sexe, sur la peur du crime. Pour l'échantillon canadien, la peur concrète de victimisation est élevée chez $45 \%$ des fermmes $(\mathrm{N}=191 / 425)$ et chez $28 \%$ des hommes $(N=108 / 392)$. La différence est encore plus grande en ce qui concerne la peur diffuse; celle-ci est élevée chez $57 \%$ des femmes $(\mathrm{N}=241 / 425)$ comparativement à chez seulement $17 \%$ des hommes $(\mathrm{N}=68 / 392)$. Pour ce qui est de la relation "peur/âge ", il ressort que la peur concrète diminue progressivement avec l'âge ; elle touche $38 \%$ des gens de 30 ans et moins $(\mathrm{N}=90 / 237), 37 \%$ de ceux qui sont âgés de 31 à 60 ans $(N=149 / 398)$ et $33 \%$ de ceux qui ont 60 ans et plus $(N=60 / 182)$. Quant à la peur diffuse, elle suit une toute autre courbe ; elle est élevée chez $40 \%$ des jeunes de 30 ans et moins $(\mathrm{N}=94 / 237)$, chez $33 \%$ des personnes âgées de 31 à 60 ans $(N=131 / 398)$ et chez. $46 \%$ des personnes de 61 ans et plus $(N=84 / 132)$. Les relations qui 
existent entre l'âge et les différents types de peur apparaîssent au Tableau II et font voir des similitudes étonnantes avec les distributions des données américaines, fournies par le Rapport Figgie.

TABLEAU II

Impact de l'àge sur la peur concrète et sur la peur diffuse, selon les données de la recherche canadienne effectuée par le G.R.A.C. (1982)

\begin{tabular}{lcccc|ccccr}
\hline & \multicolumn{4}{c|}{ Peur concrète } & \multicolumn{4}{c}{ Peur diffuse } \\
& \multicolumn{2}{c}{ Moins de } & \multicolumn{2}{c|}{61 ans et } & \multicolumn{2}{c}{ Moins de } & \multicolumn{2}{c}{61 ans et } \\
& $\mathrm{Nb}$ & $\%$ & \multicolumn{2}{c}{ plus } & \multicolumn{2}{c}{61 ans } & \multicolumn{2}{c}{ plus } \\
Peur élevée & 239 & $(38)$ & 60 & $(33)$ & 225 & $(35)$ & 84 & $(46)$ \\
Peur modérée ou faible & 396 & $(62)$ & 122 & $(67)$ & 410 & $(65)$ & 98 & $(54)$ \\
\cline { 2 - 11 } & Total & 635 & $(100)$ & 182 & $(100)$ & 635 & $(100)$ & 182 & $(100)$ \\
\hline
\end{tabular}

Nous voyons donc que, chez les Canadiens, une peur élevée de victimisation atteint une plus grande proportion de gens de moins de 61 ans $(38 \%)$ que de gens plus âgés (33\%). D'après nos chiffres, cette différence qui se dessine en regard de l'âge s'explique, en grande partie, par le sexe. En effet, en considérant les femmes et les hommes séparément, on dénote que $47 \%$ des femmes de moins de 61 ans témoignent d'une peur d'être victime de vol ou d'assaut contre $38 \%$ des femmes plus âgées. Chez les hommes, il n'y a pratiquement pas de différence selon l'âge : il n'y a que $28 \%$ des hommes de moins de 61 ans et que $27 \%$ de ceux qui ont 61 ans et plus qui manifestent un haut degré d'appréhension à l'égard d'une victimisation éventuelle.

Dans le cas de la peur diffuse, ce sont les personnes âgées (46\%) plus que les autres ( $35 \%)$, qui font montre d'une plus grande insécurité. Ce sentiment élevé de crainte diffuse touche la majorité des femmes, et, parmi elles, plus des deux tiers des femmes âgées (68\% de celles qui ont 61 ans et plus) et un peu plus de la moitié des autres (53\% des femmes de moins de 61 ans). Chez les hommes, le sentiment élevé d'insécurité demeure constant et bas, selon les groupes d'âge : $18 \%$ des 61 ans et plus et 17\% des moins de 61 ans.

Ces résultats semblent logiques et répondent aux habitudes de vie des divers groupes d'âge. Les jeunes, plus mobiles et plus aventureux, sortent davantage que les personnes âgées, que ce soit de jour ou de nuit, et ils fréquentent plus souvent des endroits où les risques de victimisation sont plus élevés (discothèques, bars, centre ville, quartiers marginaux, milieu de drogue). Ce qui explique qu'ils aient un plus haut niveau de peur concrète du crime. De plus, dans le groupe 
d'âge des 30 ans et moins, les jeunes filles sont beaucoup plus nombreuses que les garçons $(60 \%$ et $22 \%$ respectivement) à craindre d'être victimes d'un délit ou d'un crime. Cette différence vient du fait que l'on retrouve près de deux fois et demie plus de personnes, parmi les jeunes filles (29\%) que parmi les jeunes hommes (12\%), qui pensent " souvent " ou " très souvent " que quelqu'un pourrait entrer dans leur maison et les attaquer. Chez les personnes âgées (61 ans et plus) la peur d'être volé est moins répandue que chez les jeunes (34\% contre 40\%) alors que la crainte d'être attaquée reste stable chez les femmes (28\%) et chez les hommes (15\%).

Par ailleurs, la peur diffuse paraît frapper, de façon privilégiée, les personnes âgées. Et, de fait, cette peur semble liée à la fois au sexe et à l'âge. Les deux variables seraient cumulatives et agiraient, l'une et l'autre, d'une manière conjointe sur le sentiment d'insécurité. Il est certain, nous l'avons vu, que cette insécurité se révèle beaucoup plus fréquemment chez les femmes (68\%) que chez les hommes (18\%). Mais il reste que pour certains éléments de l'indice de la peur diffuse, une césure - provoquée par l'âge - se manifeste autant chez les sujets de l'un ou l'autre sexe, bien qu'à des degrés divers. À cet égard, le facteur le plus éloquent est la crainte de sortir seul le soir par peur du crime. Le Tableau III indique les relations qui, en fonction de cette crainte, se nouent entre l'âge et le sexe.

\section{TABLEAU III}

Relations entre l'âge et le sexe en fonction du degré élevé de crainte qu'ont les gens de sortir seuls le soir, à cause de la peur du crime

\begin{tabular}{lll|lr|rrr}
\hline \multicolumn{2}{c}{$\begin{array}{c}\text { Proportions de femmes et d'hommes qui évitent " souvent " ou " très souvent " de } \\
\text { sortir seuls le soir par peur du crime }\end{array}$} \\
\hline Groupes d'âge & \multicolumn{2}{c|}{ Femmes } & \multicolumn{2}{c|}{ Hommes } & \multicolumn{2}{c}{ Total } \\
& $\mathrm{Nb}$ & $\%$ & $\mathrm{Nb}$ & $\%$ & $\mathrm{Nb}$ & $\%$ \\
30 ans et moins & $43 / 111$ & 39 & $9 / 126$ & 7 & $52 / 237$ & 22 \\
30 à 60 ans & $74 / 211$ & 35 & $12 / 187$ & 6 & $86 / 398$ & 22 \\
61 ans et plus & $62 / 103$ & 60 & $13 / 79$ & 16 & $75 / 182$ & 41 \\
\hline
\end{tabular}

Ce que nous indique le Tableau III c'est que, dans le groupe d'âge des 30 ans et moins comme dans celui des personnes âgées de 30 à 60 ans, il n'y a pas de changement significatif dans la proportion de femmes ( 39 et $35 \%$ ) et d'hommes ( 7 et $6 \%$ ) qui évitent de sortir le soir par peur du crime. Cependant, chez les personnes du troisième âge (61 ans et plus), le pourcentage d'individus craintifs double presque puisqu'il passe, chez les femmes, à $60 \%$ et, chez les hommes, à $16 \%$. Ce qui montre, comme l'ont affirmé la plupart des sondages basés uni- , 
quement sur cet aspect de la crainte, que l'âge influe d'une certaine façon sur la peur diffuse du crime.

Considérant maintenant les autres aspects du sentiment d'insécurité, nous observons qu'ils ne varient presque pas, chez la femme, avec l'âge. Ainsi, quelque soit l'âge, $15 \%$ des femmes se sentent en insécurité dans leur quartier, environ $60 \%$ d'entre elles évitent d'effectuer certaines choses par peur du crime, et près de $30 \%$ déclarent ne pas aimer fréquenter certains lieux. Chez les hommes, un haut niveau d'insécurité ne frappe que 5\% de la population, peu importe les groupes d'âge. Par ailleurs, les attitudes d'évitement diminuent avec l'âge. La proportion des hommes qui se privent de certaines activités par peur du crime passe de $29 \%$ (chez les 30 ans et moins) à $20 \%$ (chez les 61 ans et plus), et celle de ceux qui évitent de fréquenter certains endroits tombe de $23 \%$, chez les plus jeunes, à $10 \%$, chez les plus vieux.

Reste à savoir si ce sentiment d'insécurité, cette peur diffuse, que ressentent davantage les personnes âgées, découle du phénomène criminel ou du processus de vieillissement, ou des deux à la fois. Mais làdessus, nous n'avons pas encore de données. Et c'est sans doute dans cette direction que devraient s'orienter les recherches portant sur la peur du crime chez les personnes âgées ; ce qui permettrait d'effectuer certaines jonctions entre les approches gérontologiques et criminologiques.

Les questions auxquelles, entre autres, il faudrait tenter de répondre sont les suivantes : existe-t-il une corrélation entre la peur du crime et les autres sources d'inquiétude qui peuvent surgir avec l'âge : crainte d'être seul, de ne pas disposer de ressources financières suffisantes, d'être malade ou de mourir ? La peur différentielle des femmes et des hommes âgés, que nous avons observée, tire-t-elle ses racines de conditions de vie différentes? L'homme, par son travail, a-t-il pu mieux domestiquer les sources d'angoisse et mieux évaluer les risques réels de victimisation? Pour la femme, surtout celle qui n'a pas travaillé, l'isolement social, auquel vient s'ajouter, dans bien des cas, la solitude, ne sont-elles pas responsables d'une crainte plus élevée et, par conséquent, d'un plus grand repli sur soi ? Dans quelle mesure, la force physique, la capacité de se défendre, le sentiment de pouvoir contrôler les risques de victimisation, sont-ils des facteurs qui influencent l'importance et l'ampleur de la peur du crime chez les personnes âgées ? Ce sont là, pour l'instant, autant de questions qui demeurent sans réponse. 


\section{EST-CE PARCE QU'ELLES ONT PLUS PEUR QUE LES PERSONNES ÂGÉES SONT PLUS PUNITIVES?}

Dans le discours public, la peur du crime est étroitement liée à la punitivité. Et dans ce sens, comme le souligne Kegels (1982) elle répond à une forme d'idéologie de la sécurité ; idéologie qui débouche irrémédiablement sur des campagnes qui visent à renforcer la sécurité personnelle des citoyens contre le crime. Ce qui a pour effet, bien souvent, d'augmenter encore plus l'insécurité elle-même ; laquelle, à son tour, amplifie la peur. Ce cercle vicieux ne semble pouvoir être brisé, pour certains groupes de pressions (dont les policiers), que par un recours à une répression plus forte.

Or, dans la réalité, la peur du crime, chez les individus, ne paraît pas entretenir de liaison spécifique avec la punitivité. Les personnes qui ont peur d'être victimes n'ont aucune tendance particulière à exiger des sentences plus sévères pour les criminels. Preuve en est qu'aux États-Unis les Noirs et les femmes, qui sont plus craintifs vis-à-vis du crime, sont moins punitifs que les Blancs et les hommes (Stinchcombe et al., 1980). Le Rapport Figgie parvient à cette même conclusion :

Nos analyses révèlent que le soutien apporté à des mesures spécifiquement punitives est indépendant autant du niveau de la peur concrète que de celui de la peur diffuse. En fonction des deux types de peur, il y a des proportions presque égales de personnes craintives et non craintives qui sont en faveur de la peine de mort ou en faveur de longues sentences d'emprisonnement (1980, p. 127).

L'étude effectuée par le Groupe de recherche sur les attitudes envers la criminalité (G.R.A.C., 1982), fait aussi état d'un manque de corrélation significative entre, d'une part, la punitivité et, d'autre part, aussi bien la peur concrète de victimisation (gamma $=, 07$ ) que le sentiment diffus d'insécurité (gamma $=, 06$ ). La même absence de lien se vérifie pour le sexe (gamma $=, 01$ ). Par contre, les données montrent assez clairement que la punitivité varie en fonction de l'âge (gamma $=, 17$ ) :4 ainsi nous constatons qu'il y a $54 \%$ de personnes punitives, parmi les personnes âgées (61 ans et plus), alors qu'il n'y en a que $35 \%$, chez les plus jeunes (30 ans et moins).

Les résultats de la recherche de Figgie indiquent que les personnes âgées ( 60 ans et plus) sont plus nombreuses à être en faveur de la peine de mort (76\%) que les autres $(71 \%)$ et que, surtout, les moins de 30 ans $(67 \%)$. Les résultats attestent, de plus, que les femmes $(68 \%)$ sont moins que les hommes (76\%) favorables à la peine capitale. Mal- 
heureusement, aucune investigation, par âge et par sexe, n'a été faite pour les autres questions portant sur les attitudes envers la sévérité des sentences accordées par les tribunaux ou, encore, envers celles vis-àvis des longues peines d'emprisonnement. De telle sorte qu'il nous est difficile de vérifier l'ampleur et les modalités de la punitivité selon les différents groupes d'âges ou, de façon simultanée, selon l'âge et le sexe.

Nous avons procédé à ces analyses pour les données de la recherche canadienne. Ainsi, parmi les personnes âgées (61 ans et plus), nous avons relevé qu'il y en a $80 \%$ qui jugent que les sentences données par la cour ne sont pas assez sévères, contre $59 \%$ chez les plus jeunes; qu'il y en a $63 \%$ (comparés à $56 \%$ chez les 30 ans et moins) qui ne pensent pas qu'il soit inhumain de garder des criminels en prison pour vingt-cinq ans et plus; qu'il y en a $75 \%$ qui ne désirent pas que la vie carcérale soit améliorée (contre $65 \%$ chez les jeunes); qu'il y en a $65 \%$ qui sont pour la peine de mort, ce qui est 1,7 fois plus que chez les jeunes ( $38 \%$ d'entre eux sont favorables à la peine capitale). Pour chacune de ces quatre questions les personnes âgées se montrent plus punitives que les autres. Des corrélations fortement significatives existent entre l'âge et :

1. le désir de sentences plus sévères (gamma $=, 33 ; p \leqslant, 0000$ )

2. le souhait de ne pas voir la vie en prison devenir plus facile (gamma $=, 22 ; p \leqslant, 0000$ )

3. l'attitude favorable à la peine de mort (gamma $=, 29 ; \mathrm{p} \leqslant, 0000$ )

TABLEAU IV

Impacts du sexe et de l'âge sur la punitivité d'un échantillon canadien (G.R.A.C., 1982)

\begin{tabular}{lcccc|ccccr}
\hline & \multicolumn{4}{c|}{ Femmes } & \multicolumn{4}{c}{ Hommes } \\
$\begin{array}{l}\text { Degré de } \\
\text { punitivité }\end{array}$ & \multicolumn{2}{c}{$\begin{array}{c}\text { Moins de } \\
\text { 61 ans }\end{array}$} & \multicolumn{2}{c|}{61 ans et } & \multicolumn{2}{c}{ Moins de } & \multicolumn{2}{c}{61 ans et } \\
& $\mathrm{Nb}$ & $\%$ & $\mathrm{Nb}$ & $\%$ & $\mathrm{Nb}$ & $\%$ & $\mathrm{Nb}$ & $\%$ \\
Punitivité élevée & 144 & $(45)$ & 56 & $(54)$ & 143 & $(46)$ & 42 & $(53)$ \\
$\begin{array}{l}\text { Punitivité modérée ou } \\
\text { faible }\end{array}$ & 178 & $(55)$ & 47 & $(46)$ & 170 & $(54)$ & 37 & $(47)$ \\
Total & 322 & $(100)$ & 103 & $(100)$ & 313 & $(100)$ & 79 & $(100)$ \\
\hline
\end{tabular}

Par contre, la relation entre l'âge et le fait de trouver inhumaines les peines de prison de 25 ans et plus n'est pas significative (gamma $=, 06 ; p \leqslant, 08$ ). Ces quatre questions, dont le coefficient de consistance est élevé (alpha $=, 66$ ), ont servi à l'élaboration d'une échelle de punitivité. Et c'est en fonction de cette échelle que nous 
avons distribué, au Tableau IV, notre échantillon en différenciant à la fois l'âge et le sexe.

Les distributions qui apparaissent au Tableau IV attestent qu'il n'y a aucun lien entre la punitivité et le sexe, puisque les distributions sont identiques pour les hommes et pour les femmes. Ayant noté, plus haut, que plus de femmes que d'hommes affichaient de hauts niveaux de peur de victimisation et de sentiment d'insécurité, la similitude des distributions de la punitivité, que nous relevons ici, prouve que cette dernière n'est guère influencée par la peur du crime. Si ce n'était le cas, le pourcentage de femmes témoignant d'une punitivité élevée serait supérieur à celui des hommes. Ce qui ne se vérifie aucunement. Pour l'un et l'autre sexe, la punitivité varie et augmente avec l'âge, et cela dans des proportions identiques. Les personnes âgées, prises comme groupe spécifique, se différencieraient des autres encore plus par une punitivité plus grande que par leur peur du crime.

Or, au niveau des attitudes générales, les informations recueillies, auprès de nos 817 répondants canadiens, nous forcent à constater que les groupes ou les individus qui se montrent les plus punitifs sont, en même temps, ceux qui sont les moins libéraux. En effet, dans notre échantillon, les personnes qui désirent que le système pénal soit plus dur envers les délinquants sont, dans une large mesure, celles qui ont une vision du monde plus rigide, plus conservatrice, moins tolérante. Par exemple, plus les gens se montrent sévères :

1. plus ils sont en faveur ( $68 \%$ d'entre eux contre $48 \%$ des moins punitifs) d'un gouvernement plus autoritaire pour mieux assurer l'ordre public (gamma $=, 31 ; p \leqslant, 0000$ )

2. plus ils pensent ( $64 \%$ contre $49 \%$ ) que les femmes qui ont des enfants doivent rester à la maison pour s'en occuper (gamma $=, 22 ; \mathrm{p} \leqslant, 0000)$

3. plus ils estiment (85\% d'entre eux contre $72 \%$ de ceux qui ont un degré modéré ou faible de punitivité) que, si ça va mal aujourd'hui, c'est parce que la famille ne joue plus le même rôle qu'autrefois (gamma $=, 28 ; \mathrm{p} \leqslant, 0000$ )

4. plus ils sont d'avis (à $58 \%$ comparés à $50 \%$ pour les autres) que, actuellement, les gens ne respectent plus rien (gamma $=, 14 ; \mathrm{p} \leqslant, 0002$ )

5. plus nombreux ils sont ( $47 \%$ contre $26 \%$ ) à refuser que, dans notre société les homosexuels soient acceptés comme tout le monde (gamma $=, 36 ; \mathrm{p} \leqslant, 0000$ ).

En conséquence, il semble donc logique de retrouver parmi les gens punitifs et conservateurs, ceux qui sont - culturellement - les plus ancrés dans le passé, dans les valeurs et les institutions ancestra- 
les, ceux qui sont les plus en faveur du maintien de l'ordre établi et des droits acquis. La plus grande punitivité des personnes âgées résulterait, dans une large mesure, non pas de leur plus grande peur du crime mais de leur vision du monde plus conservatrice. Ce qui nous conduit à cette conclusion c'est que, entre l'âge et l'axe "conservateur/ libéral " (défini par les cinq éléments qui viennent d'être énumérés) la corrélation s'avère extrêmement forte (gamma $=, 48)$ : on dénombre $37 \%$ d'individus plutôt " conservateurs " parmi ceux qui ont 30 ans et moins; $63 \%$ parmi les personnes de 31 à 60 ans; et $84 \%$ parmi les gens âgés de 61 ans et plus. D'autre part, il y a également une corrélation positive très forte entre le conservatisme et la punitivité (gamma $=, 34):$ parmi les gens qui démontrent un degré élevé de punitivité il y en a $56 \%$ qui sont plutôt conservateurs et $34 \%$ qui sont plutôt libéraux (voir le Rapport final, G.R.A.C., 1982). Ce qui ressort de ces chiffres, c'est qu'il est indéniable que toute recherche, portant sur les attitudes des personnes âgées face à la criminalité et face à la justice pénale, ne peut être complète sans une étude approfondie à la fois de leurs conditions de vie, et à la fois de leurs visions du monde.

\section{CONCLUSION}

Les recherches criminologiques, en ce qui concerne la peur du crime et la punitivité chez les personnes âgées, aboutissent à deux constations fondamentales :

1. c'est moins la crainte d'être victimes de crimes violents qu'un sentiment plus grand d'insécurité qui semble caractériser l'inquiétude qu'ont les personnes âgées face au crime ;

2. les attitudes punitives sont reliées à l'âge et se retrouvent davantage chez les personnes âgées, sans qu'il n'y ait de liens entre la punitivité et le sentiment d'insécurité.

Ces attitudes, qui définissent les façons de réagir des gens du troisième âge vis-à-vis du phénomène criminel, restent cependant très peu expliquées. Cela parce que, jusqu'à maintenant, il n'y a pas eu de recherches qui ont tenté d'analyser, d'une part, le sentiment d'insécurité en fonction du style de vie des personnes âgées et, de l'autre, la punitivité en relation avec leur vision du monde. Or, la connaissance des façons de vivre et de penser s'avère absolument nécessaire pour comprendre la nature de la peur, ressentie par les gens âgés à l'égard du crime, ainsi que le fondement de leur plus grande sévérité à l'égard des criminels. 
Pour ce qui est de la crainte de victimisation, l'enquête du G.R.A.C. montre que les crimes dont ont le plus peur les personnes de 61 ans et plus sont, pour $68 \%$ d'entre elles contre $86 \%$ des individus de 30 ans et moins, les crimes de violence. Si les personnes âgées craignent moins la violence que les jeunes, il est logique qu'elles fassent montre, proportionnellement, d'une plus faible peur concrète de victimisation. Ces perceptions différentielles des objets de crainte dépendent, sans doute, des conditions et des habitudes de vie propres aux divers groupes d'âge. Et ce sont ces conditions et ces habitudes qu'il faudrait étudier plus en profondeur pour être en mesure de mieux expliquer les attitudes. Pour parvenir à ce niveau d'analyse, il faudrait distinguer - parmi les personnes âgées - celles qui ont travaillé de celles qui n'ont jamais eu d'emploi ; celles qui sont actives de celles qui ne le sont pas ; celles qui sont seules ou malades des autres... etc. Ce sont là autant de variables, parmi d'autres tout aussi essentielles, qui décrivent les conditions de vie et qui permettent de différencier les personnes âgées selon leurs comportements ; cette différenciation devant apporter des éléments pertinents pour mieux interpréter les attitudes de peur ou d'insécurité. Il nous paraît, en tout cas, indispensable d'orienter les recherches criminologiques sur les personnes âgées selon une approche qui soit plus apte à saisir les attitudes à partir des contextes dans lesquels elles se manifestent.

La même remarque vaut également pour le schéma explicatif de la plus grande sévérité qu'expriment les personnes âgées. Aucune variable socio-démographique ou contextuelle n'explique de façon satisfaisante la corrélation positive entre l'âge et la punitivité. Nous avons $\mathrm{vu}$, précédemment, que si les personnes âgées se montrent, globalement, plus sévères c'est parce qu'une plus grande punitivité se rattache davantage à une vision " conservatrice " du monde ; vision plus répandue chez les gens du troisième âge que chez les plus jeunes. Mais d'autres facteurs peuvent intervenir. On peut supposer que la punitivité dépende des images que les gens se font et des peines et de leurs fonctions. Par exemple, chez les personnes âgées, il y a $76 \%$ de ceux qui ont 61 ans et plus qui considèrent que les prisons sont de "véritables hôtels", comparativement à $45 \%$, chez les jeunes de 30 ans et moins. Le fait de percevoir la peine d'emprisonnement comme une mesure qui ne soit pas "dure ", "pénible " ou "sévère " entraîne-t-il une plus grande punitivité?

Nous ne le croyons pas. Il paraît plus juste de penser que la punitivité est une attitude de base qui s'enracine dans la personnalité des individus, dans leurs croyance et leurs valeurs, dans leur vision du 
monde et leur idéologie. À ce titre, elle interviendrait comme un élément modulateur important qui jouerait un rôle actif fondamental, d'une part, sur les images que les gens ont du crime, du criminel et de la criminalité et, d'autre part, sur les perceptions qu'ils ont du système pénal et des façons dont celui-ci réagit au crime et au criminel. Ce sont là des hypothèses qu'il faudrait tenter de vérifier. Il s'agit là de tout un terrain d'investigation que les recherches sur les personnes âgées n'ont pas encore abordé. Et c'est peut-être selon cette perspective que devraient s'orienter les recherches futures. Peut-être alors seraient-elles en mesure d'apporter des éléments nouveaux sur l'évolution, selon les groupes d'âge, des attitudes envers le phénomène criminel et envers la justice pénale.

\section{BIBLIOGRAPHIE}

ADAMS, R., SMITH, T. (1976) : Fear of Neighborhood, National Opinion Research Center Report $127 \mathrm{C}$ on the Social Change Project, National Opinion Research Center, Chicago.

BROWN, D. (1976) : "Canada and the Noose ", Entrevue accordée par H. Mohr, in Quest, mai/juin.

BUTLER, R. (1975) : Why Survive? Being old in America, New York, Harper and Row.

CHRISTIE, N. (1974) : "Definition of Violent Behaviour ", XXIII International Course in Criminology, Maracaibo, Venezuela, 28 juillet au 3 août.

CONKLIN, J.E. (1975) : The Impact of Crime, Macmillan Publishing Co. Inc.

COOK, F.L. (1980) : Testing Claims about Criminal Victimization of the Elderly : toward Age based or Age irrelevant Policies, Paper presented at the American Society of Criminology, San Francisco (California), November 5.

COOK, F.L. (1976) : "Criminal Victimization of the Elderly : A new national problem?" in E.C. Viano, Victims and Society, Visage Press, Washington.

COOK, F.L. \& COOK, T.D. (1976) : " Evaluating the Rhetoric of Crisis : A Case Study of Criminal Victimization of the Elderly ", Social Service Review, 50, p. 632-646.

COOK, F.L. ; SKOGAN, W.G., COOK, T.D. and ANTUNES, G.E. (1978) : " Criminal Victimization of the Elderly : The Physical and Economic Consequences ", The Gerontologist, vol. 18, $\mathrm{n}^{\circ}$ 4, août, p. 338-350.

CLEMENTE, F. and KLEIMAN, M. (1976) : "Fear of crime among the aged ", The Gerontologist, vol. 16, $\mathrm{n}^{\circ} 3$, p. 207-210.

CUNNINGHAM, C. (1975) : The Pattern of Crime against the Aging : The Kansas City Study. Speech to the National Conference on Crime against the Elderly, Washington, juin 5.

FIGGIE, H.E. (1980) : The Figgie Report on Fear of Crime : America Afraid, Part 1 : The General Public, A-T-O- Inc., Ohio.

GOLDSMITH, J. and GOLDSMITH, S.S. (eds) (1976) : Crime and the Elderly, Lexington (Mass.), Lexington Books.

GOLDSMITH, J. and THOMAS, N.E. (1974) : "Crimes against the Elderly : a continuing national crisis", Aging, juin, p. 10-13. 
Groupe de Recherche sur les attitudes envers la criminalité (1982) : Les Attitudes du public canadien envers les politiques criminelles, Rapport final, Université de Montréal, C.I.C.C., 300 p.

GROSSMAN, D.A. (1977) : Reducing the Impact of Crime against the Elderly : A Survey and Appraisal of Existing and potential programs, Hollywood (Calif.), Media Five, $42 \mathrm{p}$.

HAHN, P.H. (1976) : Crimes against the Elderly : A Study in Victimology, Santa Cruz, Davis Publication.

KATZENBACH, N. de B. et al. (1967) : The Challenge of Crime in a Free Society, Washington, U.S. Gov. Printing Office.

KEGELS, M.L. (1982) : "Actualités bibliographiqes : Le crime, puisqu'il faut l'appeler par son nom... La "peur du crime ", Déviance et Société, trim./juin, vol. VI, $n^{\circ} 2$, p. 209-221.

LAWTON, M.P. (1980) : "Victimization and the Fortitude of the Aged ", Aging Care and Services, in press.

LAWTON, M.P. and JAFFEE, S. (1980) : "Victimization and Fear of Crime in Elderly Public Housing Tenants ", J. of Gerontology, 35, septembre, p. 768-779.

LAWTON, M.P., MAHEMOW, L., YAFFE, S. and FELDMAN, S. (1976) : " Psychological Aspects of Crime and Fear of Crime", in Goldsmith, J. and Goldsmith, S. (Eds.), Crime and the Elderly, Lexington (Mass.), Heath.

LEVITAN, S. (1976) : Programs in Aid of the Poor, Johns Hopkins Univ. Press., Baltimore.

LINDQUIST, J.H. and DUKE, J.M. (1982) : "The Elderly Victim at Risk ", Criminology, vol. $20, \mathrm{n}^{\circ} 1$, mai, p. 115-127.

LOGAN, M.M. (1979) : "Crime against the Elderly : Cruel and Unusual Punishment ", Victimology, vol. 4, $\mathbf{n}^{\circ}$ 1, p. 129-132.

SKOGAN, W.G. (1980) : Adjusting Rates of Victimization for Exposure to Risk, to understand the Crime Problems of the Elderly. Paper presented at the American Society of Criminology, San Francisco (Calif.), novembre.

SKOGAN, W.G. (1977) : " Public Policy and the Fear of Crime in Large American Cities ", in Gardiner, J.A. (Ed), Public Law and Public policy, New York, Praeger.

STINCHCOMBE, L.A., et al. (1980) : Crime and Punishment Changing Attitudes in America, Jossey-Boss Publishers, San Francisco - Washington - London.

SUNDEEN, R. and MATHIEU, J. (1976a) : " The Fear of Crime and its Consequences among Elderly in three Urban Communities ", Gerontologist, 16 (3), p. 211-219.

SUNDEEN, R. and MATHIEU, J. (1976b) : "The Urban Elderly : Environments of fear ", in Goldsmith, J. and Goldsmith, S. (eds), Crime and the Elderly, Lexington (Mass.), Heath.

U.S. CONGRESS, House (1977) : "Select Committee on Aging ", in Search of Security : A National Perspective on Elderly Crime Victimization, USGPO, Washington.

U.S. CONGRESS, Senate (1972) : Special Committee on Aging Hearing before the Subcommittee on Housing for the Elderly, $92^{\mathrm{e}}$ Cong., $1^{\mathrm{re}}$ sess., octobre.

YIN, PETER P. (1980) : " Fear of Crime among the Elderly : Some Issues and Suggestions ", in Social Problems, 27, p. 492-504. 